\title{
Necrosis foliar; nuevo síntoma asociado a la pudrición de la raíz de chile (Capsicum annuum) en Durango y Zacatecas, México
}

\section{Foliar necrosis; new symptom associated to root rot of pepper (Capsicum annuum) in Durango and Zacatecas, Mexico}

\author{
Rodolfo Velásquez-Valle, Luis Roberto Reveles-Torres
}

Campo Experimental Zacatecas, INIFAP, km. 24.5 Carretera Zacatecas, Fresnillo, Calera de V. R., Zacatecas, México. CP 98500.

Luis Roberto Reveles Torres, e-mail: reveles.roberto@inifap.gob.mx

\section{RESUMEN}

Antecedentes: Uno de los principales cultivos hortícolas en Durango y Zacatecas, México es el chile (Capsicum annuum); el cual es afectado por enfermedades foliares y del suelo. Desde la temporada de cultivo 2016 un nuevo síntoma, necrosis foliar fue observado afectando plantas de chile en ambos Estados.

Objetivos: Identificar los patógenos asociados con esta nueva sintomatología en plantas de chile de Durango y Zacatecas, México.

Métodos: Plantas enfermas de chile que mostraban síntomas de necrosis foliar fueron colectadas en parcelas comerciales en Durango y Zacatecas, México. Los síntomas aéreos y radiculares que mostraba cada planta fueron registrados. La raíz y raicillas de las plantas muestreadas se sembró en PDA y se identificaron las colonias fungosas.

Resultados y conclusiones: La principal característica de esta sintomatología fue la presencia de una necrosis foliar. Un grupo de cinco patógenos (Phytophthora spp., Pythium spp., Fusarium spp., Rhizoctonia spp. y Verticillium spp.) habitantes del suelo fue identificado en el sistema radicular de las plantas de chile. La frecuencia de aislamiento fue mayor para Rhizoctonia spp., Fusarium spp. y Verticillium spp. que para Phytophthora spp. y Pythium spp.

Palabras clave: necrosis foliar, Phytophthora spp., Pythium spp., Fusarium spp., Rhizoctonia spp., Verticillium spp.

\section{SUMMARY}

Background: One of the toppest horticultural crops in the Mexican states of Durango and Zacatecas is pepper (Capsicum annuum), which is affected by foliar and soilborne diseases. Since the 2016 crop season a new symptom, foliar necrosis, was observed affecting pepper plants in both states.

Objectives: Identify the pathogens associated to this new symptomatology in pepper plants from Durango and Zacatecas, México.

Methods: Diseased pepper plants showing foliar necrosis were collected in commercial fields from Durango and Zacatecas, Mexico. Aerial and root symptoms shown in every plant were recorded; root and rootlets from every sampled plant were PDA-plated and fungi colonies were identified.

Results and conclusions: The main characteristic of this symptomatology was the presence of a foliar necrosis. A group of five soilborne pathogens (Phytophthora spp., Pythium spp., Fusarium spp., Rhizoctonia spp. y Verticillium spp.) was identified in the root system of pepper plants. The isolation frecuency was higher for Rhizoctonia spp., Fusarium spp., and Verticillium spp. than Phytophthora spp., and Pythium spp.

Keywords: Foliar necrosis, Phytophthora spp., Pythium spp., Fusarium spp., Rhizoctonia spp., Verticillium spp. 


\section{INTRODUCCIÓN}

Uno de los principales cultivos hortícolas en los estados de Durango y Zacatecas, México es el chile (Capsicum annuum L.); la producción de chile para secado, de los tipos Ancho, Mirasol y Pasilla, es la más importante aunque la producción de chile para consumo en fresco, del tipo Jalapeño, entre otros, es también relevante (Zegbe et al., 2012). Nuevos síntomas foliares aparecieron en parcelas comerciales de chile durante los últimos ciclos de cultivo: necrosis foliar ocasionalmente acompañada de defoliación y muerte regresiva de ramas; esta sintomatología afectaba completamente la parte aérea de las plantas afectadas sugiriendo un posible daño en el sistema radicular. Por otro lado, las plantas enfermas tendían a agruparse en manchones en las parcelas, en un comportamiento típico de las enfermedades causadas por patógenos del suelo. En esta región la marchitez o secadera, causada por Phytophthora spp., es la enfermedad más devastadora; su infección causa una distintiva lesión negra en los tallos, aunque las raíces también pueden ser infectadas, conduciendo a la marchitez de la planta (Ristaino, 2003), sin embargo, un estudio previo llevado a cabo en la misma región reveló la presencia de un grupo de patógenos (Phytophthora spp., Pythium spp., Fusarium spp., Rhizoctonia spp. y Verticillium spp.) asociadas a la raíz de plantas de chile que mostraban marchitez foliar (pérdida de turgencia; cambio de color de verde oscuro a verde opaco), defoliación prematura y maduración irregular de frutos, entre otros (Velásquez-Valle et al., 2001). Las enfermedades provocadas por patógenos del suelo no causan una sintomatología foliar específica; la infección causada por Verticillium dabliae Kleb., por ejemplo, en plantas de chile induce marchitez, clorosis y necrosis foliar, epinastia, coloración vascular, enanismo y la muerte (Goicoechea, 2006; Sanogo et al., 2008), sin embargo, uno o más de esos síntomas pueden ser compartidos por otros patógenos como Fusarium spp. y R hizoctonia spp. El manejo integrado de enfermedades está fundamentado en la correcta identificación del agente causal; el paso inicial para lograr dicha identificación es determinar una asociación entre los posibles agentes causales y la sintomatología observada, por lo tanto el objetivo de éste trabajo consistió en identificar y cuantificar la presencia de los géneros de patógenos asociados con esta sintomatología emergente en plantas de chile de Durango y Zacatecas, México.

\section{MATERIALES Y MÉTODOS}

Un número variable de plantas sintomáticas de diferentes tipos de chile fue colectado en parcelas comerciales seleccionadas al azar en los Estados señalados. El criterio de selección de plantas fue la presencia de necrosis foliar, defoliación o muerte regresiva de ramas. La presencia de cinco síntomas foliares (marchitez, enanismo, defoliación, bordes foliares con necrosis y ramas con muerte regresiva) fue registrada en cada planta colectada. La raíz y raicillas se examinaron para detectar la presencia de necrosis, descortezamiento y coloración vascular.

El sistema radicular fue separado del resto de la planta y enjuagado con agua corriente para remover el exceso de suelo; porciones de la raíz principal o raicillas se esterilizaron y sembraron en medio de cultivo PDA y se incubaron a $27-28{ }^{\circ} \mathrm{C}$ durante $72 \mathrm{~h}$. las colonias fungosas resultantes se identificaron empleando las claves taxonómicas propuestas por Watanabe (1994) y Barnett (1967).

\section{RESULTADOS Y DISCUSIÓN}

Se colectaron 84 plantas enfermas de chile en 13 parcelas comerciales en los estados de Durango (31 plantas) y Zacatecas (53 plantas), México. Las plantas colectadas pertenecían a los tipos Mirasol (64.3\%), Pasilla (27.4\%), Ancho (1.2\%) y Jalapeño (7.1\%).

El síntoma característico de la enfermedad era una lesión café en el borde de la hojas rodeada por una franja de forma variable pero distinguible por su color verde opaco que separaba a los tejidos necrosados del tejido sano en la lámina foliar que aún conservaba su color verde oscuro; el avance de la necrosis desde el borde podía afectar la hoja en un periodo de dos a tres días; las hojas con síntomas avanzados mostraban un rizado que inicia en el ápice y se hacía menos evidente hacia la base de la hoja, aunque finalmente toda la lámina foliar podía tomar una coloración café (Figura 1); todas las plantas muestreadas en el actual trabajo mostraban éste síntoma.

La marchitez foliar y la defoliación, son síntomas frecuentemente asociados con la infección de las raíces de las plantas de chile por patógenos como P. capsici, V. dabliae y Fusarium oxysporum f. sp. capsici (Ristaino, 2003; Sanogo et al., 2008; Abada y Ahmed, 2014), pero en el actual trabajo solamente el $1.2 \%$ de las plantas colectadas presentaban sínto- 


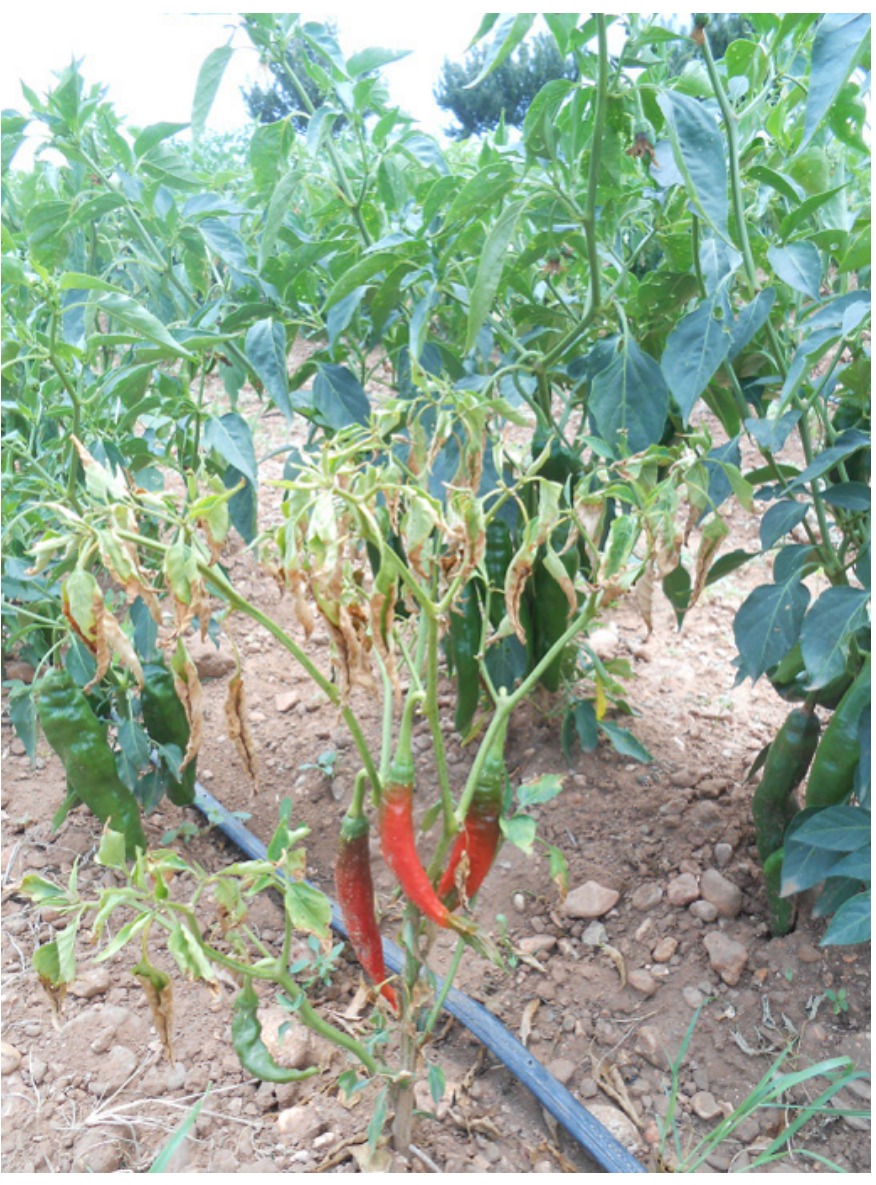

Figura 1. Planta de chile mostrando hojas con los bordes colgantes y con los bordes necrosados característicos de la sintomatología.

mas foliares típicos de marchitez (pérdida de turgencia y cambio de color verde oscuro a verde opaco sin ninguna zona de transición en la hoja) que es el síntoma foliar típico de la enfermedad tradicionalmente conocida como marchitez o secadera del chile, sin embargo, es posible que el rápido desarrollo de las lesiones necróticas (necrosis de color café en el borde de las hojas) haya ocultado los síntomas típicos de marchitez o secadera. Por otro lado, la defoliación se manifestó en $38.1 \%$ de las plantas enfermas colectadas (Tabla 1).
Otro síntoma observado fue la muerte descendente o regresiva de ramas (MRR), la cual inicia como una necrosis del ápice de las ramas; en pocos días la rama afectada puede tomar una coloración café pálido con defoliación total o parcial así como la pérdida de frutos (Figura 2). Este síntoma fue exhibido por el $23.8 \%$ de las plantas muestreadas.

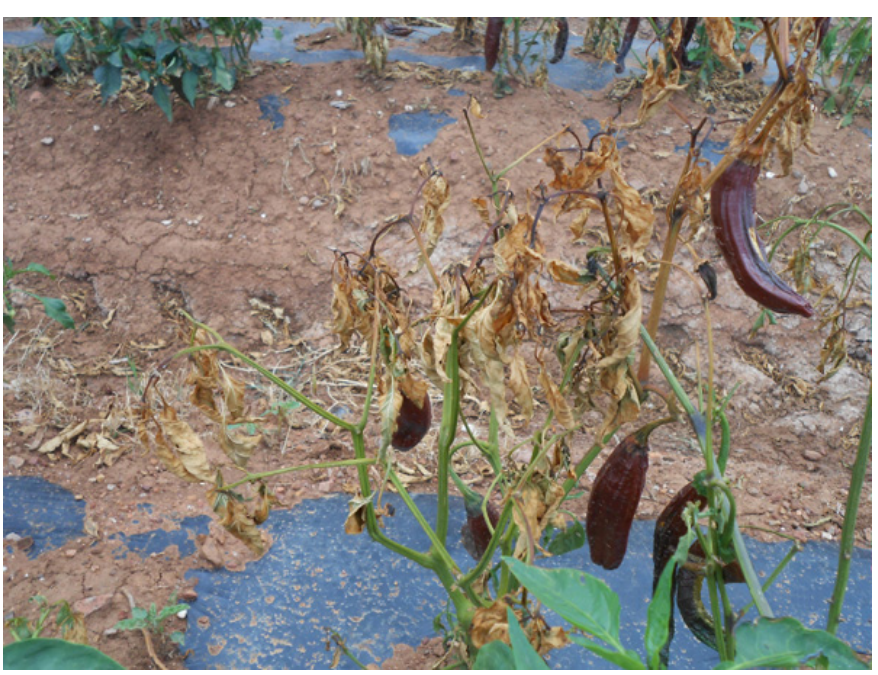

Figura 2. Planta de chile mostrando muerte descendente de ramas asociadas con una sintomatología emergente en Durango y Zacatecas, México.

El síntoma de enanismo fue registrado en solamente el $13.1 \%$ de las plantas muestreadas; la mayoría de las plantas que presentaban este síntoma se colectaron en Durango donde la incidencia de otras enfermedades bacterianas (Velásquez-Valle et al., 2014) pudiera coadyuvar en la expresión del enanismo (Tabla 1).

Es importante señalar que la necrosis foliar se presentaba afectando la planta completa sugiriendo que su origen se encontraba en el sistema radicular lo cual parece ser confirmado por el hecho de que alrededor de $94 \%$ de las plantas muestreadas manifestaba algún tipo de lesión en la raíz (pérdida de la corteza

Tabla 1. Frecuencia de expresión de síntomas aéreos y subterráneos asociados a plantas de chile con necrosis foliar

\begin{tabular}{cccc}
\hline Síntomas aéreos & Frecuencia de expresión (\%) & Síntomas subterráneos & Frecuencia de expresión (\%) \\
\hline Necrosis foliar & 98.8 & Coloración vascular & 80.9 \\
Defoliación & 38.1 & Raicillas necróticas & 26.2 \\
Muerte regresiva de ramas & 23.8 & Raicillas descortezadas & 26.2 \\
Enanismo & 13.1 & & \\
\hline
\end{tabular}


o cambio de color en los tejidos vasculares) o raicillas (necrosis o pérdida de la corteza).

Entre los síntomas subterráneos destaca la coloración café a negra del tejido vascular; cerca del $81 \%$ de las plantas muestreadas mostraban dicha coloración (Tabla 1); éste síntoma vascular ha sido reportado en plantas de chile infectadas con $F$. oxysporum f. sp. capsici y V. albo-atrum y V. dabliae (Black et al., 1991). En plantas de chile los patógenos vasculares como Verticillium spp. y Fusarium spp. y oomicetos como Phytophthora spp. interrumpen el suministro de agua por medio del xilema, y provocan la aparición de síntomas como la marchitez y defoliación (Velásquez et al., 2001; Black, 2003; Goldberg, 2003).

La necrosis y descortezamiento de raicillas se registró en el $26.2 \%$ (para cada caso) de las plantas muestreadas; es probable que después de registrarse el daño en el xilema se inicie un proceso de descomposición de las raicillas con lo que la planta no sólo se encontraría incapacitada para transportar agua y nutrientes sino que tampoco podría absorberlos (Tabla 1).

Se identificaron cinco géneros de hongos (Rhizoctonia spp., Fusarium spp. y Verticillium spp.) u oomicetos (Phytophthora spp., Pythium spp.), en las raíces de las plantas de chile muestreadas en el actual trabajo; todos esos patógenos habían sido previamente mencionados en asociación con plantas enfer- mas de chile en diferentes regiones de México (Velásquez-Valle et al., 2001; Durán-Ortiz et al., 2001; Pérez-Acevedo et al., 2017).

Se detectaron e identificaron uno o más patógenos en todas las parcelas de chile muestreadas. La frecuencia de aislamiento de patógenos fue variable; Rhizoctonia spp. y Fusarium spp. fueron identificados en todas las parcelas muestreadas; VerticiIllium spp. fue encontrado en 10 de las 13 parcelas visitadas. Los oomycetos Phytophthora spp. y Pythium spp. fueron identificados en solamente tres y cinco parcelas respectivamente.

Los hongos Rhizoctonia spp., Fusarium spp. y Verticillium spp. fueron identificados en 76.2 , 36.9 y $21.4 \%$ de las plantas muestreadas en tanto que los oomicetos Phytophthora spp. y Pythium spp. se detectaron en 3.6 y $8.3 \%$ del total de plantas muestreadas. A nivel de parcela, la frecuencia de aislamiento varió de 50 a $100 \%$ para Rhizoctonia spp. (promedio $81.2 \%$ ); de 11.1 a $80 \%$ para Fusarium spp. (promedio 40.6); y de 14.3 a $66.6 \%$ en el caso de Verticillium spp (promedio 25.2\%). Menores porcentajes de aislamiento se obtuvieron con Phytophthora spp. (6.2 a $20 \%$ ) (promedio $8.5 \%$ ) y Pythium spp. (11.1 a $33.3 \%$ ) (promedio $8.5 \%$ ) (Tabla 2).

La frecuencia de aislamiento de esos patógenos obtenida en este trabajo discrepa de los resultados previamente reportados (Velásquez-Valle et al., 2001), aunque la sintomatología mencio-

Tabla 2. Aislamiento de patógenos del sistema radicular de plantas de chile colectadas en Durango y Zacatecas con síntomas de necrosis foliar

\begin{tabular}{cccccc}
\hline & \multicolumn{5}{c}{ Aislamiento (\%) } \\
\cline { 2 - 6 } Parcela & Rhizoctonia spp. & Phytophthora spp. & Pythium spp. & Fusarium spp. & Verticillium spp. \\
\hline 1 & 70 & 10 & 20 & 30 & 40 \\
2 & 56.2 & 6.2 & 12.5 & 43.7 & 18.7 \\
3 & 50 & 0 & 0 & 50 & 25 \\
4 & 100 & 0 & 33.3 & 66.6 & 33.3 \\
5 & 60 & 0 & 0 & 80 & 60 \\
6 & 100 & 0 & 0 & 66.6 & 66.6 \\
7 & 100 & 0 & 0 & 28.6 & 0 \\
8 & 50 & 0 & 0 & 66.6 & 0 \\
9 & 100 & 0 & 0 & 16.6 & 16.6 \\
10 & 100 & 0 & 0 & 14.3 & 14.3 \\
11 & 100 & 0 & 33.3 & 33.3 & 33.3 \\
13 & 80 & 20 & 0 & 20 & 20 \\
\hline
\end{tabular}


nada en ese estudio fue la típicamente asociada con la marchitez (pérdida de turgencia foliar y cambio de color de verde oscuro a verde opaco), mientras que en el presente trabajo el síntoma característico fue la necrosis del borde de las hojas. Las principales diferencias entre ambos trabajos, además de la sintomatología, incluyen el aumento en la frecuencia de aislamiento de Rhizoctonia spp. de 31.6 a $81.2 \%$ y de Verticillium spp. 2.5 a $25.2 \%$ para los estudios del año 2001 y del actual respectivamente; también la frecuencia de aislamiento de Pythium spp. y Fusarium spp. se incrementó en el actual trabajo. El caso contrario se registró con el oomyceto Phytophthora spp. cuya frecuencia de aislamiento descendió de 14.1 en 2001 a $2.8 \%$ en este estudio. Algunos factores como la fecha de muestreo, parcelas muestreadas, variaciones climáticas, etc, dificultan la comparación entre ambos trabajos, sin embargo el propósito de esta comparación es mostrar que la variación en resultados también puede deberse a que se trate de diferentes enfermedades.

Al analizar la frecuencia de aislamiento de esos patógenos por entidad (Durango y Zacatecas), independientemente del tipo de chile, se encontró que el orden de detección de los patógenos fue similar (Rhizoctonia spp., Fusarium spp., Verticillium spp., Pythium spp. y Phytophthora spp.) en ambos Estados. Sin embargo, la frecuencia de aislamiento de Fusarium spp., Verticillium spp., Pythium spp. y Phytophthora spp. fue siempre mayor en las plantas de Zacatecas; sólo en el caso de Rhizoctonia spp. la frecuencia de aislamiento resultó mayor en Durango que en Zacatecas (Tabla 3).
Tabla 3. Frecuencia de aislamiento de cinco patógenos radiculares de chile en Durango y Zacatecas

\begin{tabular}{lcc}
\hline & \multicolumn{2}{c}{ Entidad } \\
\cline { 2 - 3 } Patógeno & Durango & Zacatecas \\
\hline Rhizoctonia spp. & $83.9^{x}$ & 71.7 \\
Fusarium spp. & 32.2 & 39.6 \\
Verticillium spp. & 9.7 & 28.3 \\
Pythium spp. & 3.2 & 11.3 \\
Phytophthora spp. & 0 & 5.7 \\
\hline
\end{tabular}

× Porcentaje de aislamiento

Dentro del grupo de síntomas subterráneos observados en este tipo de plantas, la coloración vascular resultó el de mayor frecuencia por lo que se trató de relacionarlo con el aislamiento de los patógenos o combinaciones de ellos más frecuentes; cuando se identificó solamente un patógeno, (Rhizoctonia spp., Fusarium spp., o Verticillium spp.) el síntoma subterráneo más frecuente resultó ser la coloración vascular, con porcentajes de detección de 83.8, 85.7 y 75\% respectivamente. Una tendencia similar se advierte cuando se revisan las interacciones entre dos o más de esos patógenos, con la excepción de la interacción Fusarium spp. + Verticillium spp. en donde el porcentaje de detección de coloración vascular fue menor que el de raicillas necróticas y descortezadas aunque es posible que el resultado se deba a un reducido número de muestras (tres) con esa interacción (Tabla 4).

Tabla 4. Expresión de síntomas radiculares asociados con el aislamiento de Rhizoctonia spp., Fusarium spp. y Verticillium spp. en raíces de plantas de chile

\begin{tabular}{lccc}
\hline & \multicolumn{3}{c}{ Síntoma } \\
\cline { 2 - 4 } Patógeno & Raicillas necróticas & Coloración vascular & Raicillas descortezadas \\
\hline Rhizoctonia spp. & $32.4^{\times}$ & 83.8 & 24.3 \\
Fusarium spp. & 0 & 85.7 & 14.3 \\
Verticillium spp. & 0 & 75 & 25 \\
Rhizoctonia spp. + Fusarium spp. + Verticillium spp. & 60 & 80 & 60 \\
Rhizoctonia spp. + Verticillium spp. & 25 & 75 & 25 \\
Fusarium spp. + Verticillium spp. & 66.6 & 33.3 & 66.6 \\
Rhizoctonia spp. + Fusarium spp. & 16.7 & 100 & 16.7 \\
\hline
\end{tabular}

×Frecuencia de expresión de síntomas (\%). 
Tabla 5. Expresión de síntomas aéreos asociados con el aislamiento de Rhizoctonia spp., Fusarium spp. y Verticillium spp. en raíces de plantas de chile

\begin{tabular}{lccc}
\hline & \multicolumn{3}{c}{ Síntoma } \\
\cline { 2 - 4 } Patógeno & Enanismo & Defoliación & Muerte regresiva de ramas \\
\hline Rhizoctonia spp. & $29.4^{\times}$ & 58.8 & 35.3 \\
Fusarium spp. & 0 & 85.7 & 42.8 \\
Verticillium spp. & 0 & 100 & 50 \\
Rhizoctonia spp. + Fusarium spp. + Verticillium spp. & 0 & 75 & 25 \\
Rhizoctonia spp. + Verticillium spp. & 0 & 100 & 50 \\
Fusarium spp. + Verticillium spp. & 0 & 100 & 66.6 \\
Rhizoctonia spp. + Fusarium spp. & 25 & 75 & 75 \\
\hline
\end{tabular}

×Frecuencia de expresión de síntomas (\%).

La frecuencia de detección de síntomas aéreos (enanismo, defoliación y MRR) diferentes a la necrosis foliar, indicó que la defoliación fue el síntoma más común independientemente del patógeno (tomando en cuenta únicamente a Rhizoctonia spp., Fusarium spp., y Verticillium spp.), o combinación de ellos; es oportuno notar que el síntoma de enanismo sólo se produjo cuando Rhizoctonia spp. se encontraba presente individualmente o en interacción con Fusarium spp. (Tabla 5).

Al agrupar las plantas de chile sintomáticas por los tipos de chile (se consideraron solamente los tipos Mirasol y Pasilla por tener un mayor tamaño de muestra, 54 y 23 plantas respectivamente), encontrándose que no hubo cambio en el orden de los patógenos identificados (Rhizoctonia spp., Fusarium spp., Verticillium spp., Pythium spp. y Phytophthora spp.) para ambos tipos de chile, aunque la frecuencia de aislamiento de Rhizoctonia spp., Fusarium spp., Pythium spp. y Phytophthora spp. fue superior en las plantas del tipo Pasilla; por el contrario, la de Verticillium spp. fue mayor en las plantas del tipo Mirasol (Tabla 6).

Tabla 6. Frecuencia de aislamiento de patógenos asociados con la raíz de plantas de chile tipo Mirasol y Pasilla con síntomas de necrósis foliar

\begin{tabular}{lll}
\hline & \multicolumn{2}{l}{ Tipo de chile } \\
\cline { 2 - 3 } Patógeno & Mirasol & Pasilla \\
\hline Rhizoctonia spp. & $57.4^{\times}$ & 69.6 \\
Fusarium spp. & 33.3 & 39.1 \\
Verticillium spp. & 24.1 & 17.4 \\
Pythium spp. & 5.5 & 13.0 \\
Phytophthora spp. & 3.7 & 4.3 \\
\hline
\end{tabular}

${ }^{\times}$Frecuencia de aislamiento (\%).
Una sintomatología emergente que incluye la necrosis del borde de las hojas como el síntoma distintivo, pero ocasionalmente incluye muerte descendente de ramas, esto fue observado desde el ciclo de cultivo 2016 en parcelas comerciales de chile en los estados de Durango y Zacatecas, donde la marchitez o secadera del chile ( $P$. capsici) ha sido señalada como la principal amenaza para la producción de chile. Un grupo de patógenos del suelo (Phytophthora spp., Pythium spp., Rhizoctonia spp., Fusarium spp. y Verticillium spp.) fueron identificados tanto en la raíz principal como en raicillas de plantas de chile con necrosis foliar principalmente; sin embargo, este grupo de patógenos fue previamente mencionado como asociado con la pudrición de la raíz y marchitez o secadera (Velásquez-Valle et al., 2001), consecuentemente, es necesario continuar con los postulados de Koch para definir el papel de cada miembro de este grupo de patógenos en la producción de la sintomatología mencionada.

\section{REFERENCIAS}

Abada, K.A., M.A. Ahmed, 2014. Management Fusarium wilt of sweet pepper by Bacillus strains. American Journal of Life Sciences 2: 19-25.

Barnett, H.L., 1967. Illustrated genera of imperfect fungi. Burgess Publishing Company. Fourth Printing. Minneapolis, MN, USA.

Black, L.L., S.K. Green, G.L. Hartman, J.M. Poulos, 1991. Pepper diseases. A field guide. Asian Vegetable Research and Development Center. AVRDC Publication No. 91-347.

Black, L.L., 2003. Fusarium wilt. In: Pernezny, K., P.D. Roberts, J.F. Murphy, N.P. Goldberg (eds.), Compendium of pepper diseases. The American Phytopathological Society Press. St. Paul, Minnesota. Pp. 14-15.

Durán-Ortiz, L.J., L. Pérez-Moreno, J.R. Sánchez-Pale, V. Olalde-Portugal, 2001. Identificación de los hongos que ocasionan la "marchitez del chile" en la región del Bajío. Memorias del XXVIII Congreso Nacional de la Sociedad Mexicana de Fitopatología. 
Goicoechea, N., 2006. Verticillium-induced wilt in pepper: physiological disorders and perspectives for controlling the disease. Plant Pathology Journal 5: 258-265.

Goldberg, N.P., 2003.Verticillium wilt. In: Pernezny, K., P.D. Roberts, J.F. Murphy, N.P. Goldberg (eds.), Compendium of pepper diseases. The American Phytopathological Society Press. St. Paul, Minnesota. Pp. 21-22.

Pérez-Acevedo, C.E., J.C. Carrillo-Rodríguez, J.L. Sánchez-Servia, C. Perales-Segovia, V.R. Enriquez, Y. Villegas-Aparicio, 2017. Diagnóstico de síntomas asociados con marchitez de chile en Valles Centrales de Oaxaca. Revista Mexicana de Ciencias Agrícolas 8: 281-293.

Ristaino, J.B., 2003. Phytophthora blight. In: Pernezny, K., P.D. Roberts, J.F. Murphy, N.P. Goldberg (eds.), Compendium of pepper diseases. The American Phytopathological Society Press. St. Paul, Minnesota. Pp. 17-19.

Sanogo, S., O.I. El-Sebai, R. Sanderson, 2008. Severity of Verticillium wilt, plant growth, and spectral reflectance indices of chile pepper under periodic flooding and no-flooding conditions. HortScience 43: 414-419.
Velásquez-Valle, R., M.M. Medina-Aguilar, J.J. Luna-Ruiz, 2001. Sintomatología y géneros de patógenos asociados con las pudriciones de la raíz de chile (Capsicum annuum L.) en el norte centro de México. Revista Mexicana de Fitopatología 19: 175-181.

Velásquez-Valle, R., L.R. Reveles-Torres, J. Mena-Covarrubias, S. Salas-Muñoz, J.A. Mauricio-Castillo., 2014. Outbreak of Candidatus Liberibacter solanacearum in dried chile pepper in Durango, Mexico. Agrofaz 14: 93-98.

Watanabe, T., 1994. Pictorial atlas of soil and seed fungi. Morphologies of cultured fungi and key to species. CRC Press, Boca Raton.

Zegbe, D.J.A., J. Mena C., R.D. Valdez C., M.D. Amador R., G. Esparza F., 2012. Importancia, diversidad genética y situación actual del cultivo del chile en México. In: Zegbe, J.A., D., R.D. Valdez C. , A. Lara H. (eds.), Cultivo del chile en México. Proyecto Editorial UAZ, Zacatecas. Pp. 11-47. 